\title{
Analysis and Optimisation of IEEE 802.11 Wireless Local Area Networks
}

\author{
Anurag $\operatorname{Kumar}^{\dagger}$ \\ ECE Department, Indian Institute of Science \\ Bangalore, 560 012, India
}

\section{Extended Abstract}

In the recent years, IEEE 802.11 wireless local area networks (WLANS) have increasingly been deployed in a variety of situations, such as homes, business enterprises, academic campuses, and public places such as airports, hotels, and shopping centers. It has therefore become very important to understand the performance of such networks, and also how to effectively design, deploy and manage them. In this paper our concern is mainly with the analytical performance evaluation of WLANs. We will also consider an aspect of optimisation of such networks.

We will begin with a discussion of saturation throughput analysis of single cell WLANs. In this context the seminal contribution is due to G. Bianchi who proposed a decoupling approximation that led to a fixed point equation, the solution of which yielded an approximation to the attempt rate of any node. We show that, in fact, the fixed point equation can be established using a renewal reward argument and in a much more general setting. Further, in this setting a natural condition on the back-off sequence leads to uniqueness of the fixed point. It is possible to take the same decoupling approximation and develop a vector fixed point equation, assuming that the nodes have possibly unequal attempt rates. If a vector fixed point has unequal coordinates, then we say that it is unbalanced. The traditional approach only considered balanced fixed points. We consider the vector fixed point equation, and show that, in general, there can exist multiple unbalanced fixed points, even in those situations where there is a unique balanced fixed point. Simulations show that in situations where this happens the system exhibits significant short term unfairness, and further, the balanced fixed point analysis fails to capture the steady state performance. For the case in which the mean back-off grows multiplicatively with collisions, we establish a sufficient condition for a system to have a unique fixed point (that is also balanced).

The original IEEE 802.11 medium access control (MAC) mechanism provided no means for differentiating the channel access provided to the contending devices. A new addition to the IEEE 802.11 suite of standards, IEEE 802.11e, now provides several access differentiation mechanisms. One set of mechanisms differentiates access by permitting different devices (or queues in devices) to have different channel access

\footnotetext{
$\dagger$ This presentation will be based on joint work with the following coauthors: Eitan Altman, Munish Goyal, Vinod Kumar, Daniele Miorandi, Manoj Panda, Venkatesh Ramaiyan, and S.H. Srinivasan.
}

parameters, such as the initial back-off, the back-off multiplier, and the maximum back-off. Another, mechanism is that, after every successful transmission, nodes with a lower access priority wait for a little longer than other nodes before reinitiating their back-off and attempt processes, thus giving the higher priority class nodes a better chance to access the channel. This latter mechanism is called AIFS (arbitration interframe space). The vector fixed point analysis is extended to both these mechanisms, and we establish a condition for uniqueness of the fixed point.

For all the above access mechanisms, we also provide asymptotic results in the regime where the number of retrials is unbounded and the number of nodes goes to $\infty$. This analysis provides interesting analytical insights into the role of some of the access parameters.

Saturation throughput analysis is normally conducted to assess the "capacity" of these networks. However, it is possible to use the saturation throughput analysis directly in certain application situations. We provide a demonstration of this in the context of TCP controlled transfer of large files.

While much of the analysis discussed above is for single cell WLANs, in practice, in order to cover large spaces, WLANs comprise multiple overlapping cells. Since there are only three nonoverlapping frequency bands in the operational spectrum, in a typical WLAN deployment, cochannel WLAN cells will overlap. We develop a fixed point based saturation throughput analysis of two cells whose decoding ranges are disjoint but their carrier sense ranges completely overlap. The analysis captures an interesting unfairness that occurs in this situation. The model turns out to be similar to the one obtained for the AIFS based throughput differentiation mechanism mentioned above.

At the present time there do not appear to be systematic techniques to design and optimise WLANs. We will briefly discuss the problem of optimal association of devices to access points (APs) in an infrastructure WLAN. The problem is that there are several mobile devices, each of which can potentially connect via one of several candidate APs, at various physical layer rates. The question is: "What is an optimal association?" We formulate the problem as one of maximising, over the possible associations, the sum of the up-link utilities obtained at the devices. Thus the problem is like the optimal bandwidth sharing problem, well known in wired packet networks, with the additional complexity that the network "topology" also needs to be simultaneously obtained. We formulate the problem, discuss the issues, and provide some preliminary results. 